\title{
61 \\ Building computer tools for negotiation- The Family_Negotiator Project
}

\author{
E. Bellucci and J. Zeleznikow \\ CLAIR Project, Database Research Laboratory, \\ Applied Computing Research Institute, \\ La Trobe University, Bundoora, Victoria, Australia, 3083.
}

Phone: +61.3.9479 1003; Fax: +61.3.9479 3060;

E-mail: \{bellucci,johnz\}@latcsl.cs.latrobe.edu.au

\begin{abstract}
While Artificial Intelligence techniques such as case based reasoning and rule based reasoning have proven successful in modelling legal domains, a more flexible reasoning paradigm may be required to model the dynamics of negotiation. Hybrid systems are a possible solution. Family_Negotiator employs such an architecture, providing disputing couples with a computerised implementation to suggest possible solution paths, obtained from previous cases with similar characterisics. The system's role as a support system to family law negotiations is justified in its attempt to suggest possible outcomes at different recorded stages of negotiation. Through the hybrid reasoning techniques employed in Family_Negotiator, the functionality of the decision support system is realised.
\end{abstract}

Keywords

Australian Family Law, negotiation, hybrid systems

\section{INTRODUCTION}

Hybrid systems have become increasingly popular in developing decision support systems since they acquire the benefits of two or more Artificial Intelligence pardigms into the one system. Family law negotiation is best modelled by hybrid rule based and case based reasoning. These paradigms are complementary in their reasoning techniques, enabling modelling of two forms of knowledge representation used in family law decision support. Specifically, open textured terms can be well supported by case based reasoning, while heuristics and legislation pertaining to Family Law can be modelled successfully through rule 
based reasoning. A term is open textured if it contains questions that cannot be structured in the form of production rules or logical propositions and which require some legal knowledge on the part of the user in order to answer.

Family_Negotiator was developed as a hybrid rule based, case based reasoner, dealing in the negotiation domain of Australian Family Law. It's major aim is to act as a support tool to human negotiation, by providing a disputing couple with some guidance to enable suitable settlement. It specifically deals with the issues of child custody, determining the contents of the common pool, and deciding what percentage split of the common pool each partner receives. Solutions to these issues are retrieved by referencing several case bases, each one consisting of a collection of single arguments. The rule based reasoner aims to introduce general heuristics and law applicable to Australian Family Law.

Another system operating within the domain of family law negotiation is DEUS (Zeleznikow et. al. 1995). DEUS has been implemented using a model which represents the goals and beliefs of the opposing parties on an intelligent template. It helps mediators understand what issues are in dispute and the extent of the dispute over these issues. DEUS supports negotiation by providing an organised intelligent blackboard, whereas Family_Negotiator (Bellucci and Zeleznikow, 1996) makes use of several artificial intelligence paradigms to make practical suggestions on how to settle family law disputes.

\section{MODELLING ISSUES}

In attempting to model legal negotiation, the system developer needs to consider two aspects, what are appropriate negotiation strategies and which Artificial Intelligence paradigm should be applied in implementating these strategies.

Principled Negotiation developed under the Harvard Negotiation Project (Fisher, et. al 1994) best models the dynamic property of Australian Family Law negotiation, since this approach advocates separation of the problem from the people, a requirement that is consistent with a computer model requiring rationality among the parties. It advocates searching for mutual gains, that is, wherever interests conflict, one should come to a ruling that is independent of the beliefs of either side. It promotes a certain focus on interests in trying to change the other parties position, other than in deteriorating it into a contest of 'who will back down first', as is the case in Positional Negotiation.

Requirements of the negotiation process includes stance revision capabilities; and hence the ability to generate alternate compromising solutions. Another capability is to reason with incomplete or uncertain knowledge. A suitable Artificial Intelligence paradigm for such requirements is that of case based reasoning, since it operates by obtaining solutions from similar cases stored in its extensive case base. Since the case base can be referenced often, case based reasoning can generate several solutions, each one representing a different interpretation of the case base knowledge. There are other aspects of family law settlement that make extensive use of common law or well known heuristics. The determination of the contents of the common pool is dictated by heuristics, indicating a need for a rule based reasoning paradigm to be implemented. Hence, a hybrid rule based and case based system, employing Principled Negotiation as its negotiation strategy has been implemented in Family_Negotiator. 


\section{FAMILY_NEGOTIATOR}

Family_Negotiator as a hybrid rule based and case based reasoner incorporates the use of knowledge in the form of rules and cases throughout particular sections of the system. The system considers three distinct areas of negotiation. These are:

- Determining child custody;

- Determining the contents of the common pool;

- Determining the percentage split of the common pool each partner receives.

The system refers to 3 case bases, each one holding cases of one frame type. The basic case base holds knowledge specifically stored to provide general information about the case, such as the couple's names, the number of years of marriage, whether they have children and a list of issues this case needs to consider. This frame is of most use when the case based reasoner retrieves the contents of the 'issues' slot to help negotiate the order in which issues should be presented.

The frames used in the custody case base and the percentage split case base, which reflect arguments concerning child custody and percentage split respectively, translate to the background knowledge required to present a view. Each frame represents a single argument, its structure an adaptation of Toulmin Argument Structures (Zeleznikow and Stranieri, 1995).

Toulmin Argument Structures distinguish the data, warrant, backing and claim of a argument. Toulmin (1958) made the observation that every argument makes a declaration or insistence on the data presented. This represents the claim of the argument. Justification is given to the claim through the warrant. The warrant is necessary since it acts as a justification mechanism for the claim, given the data. The backing supports the warrant, and in legal representations, references a statute or common law.

Family_Negotiator represents an argument consistant with the above format since the structuring of an argument represents symbolically the internal workings of argumentation, and is presented in a form easily utilised by case based reasoning. Entry of the parties claim ('husband_stance' and 'wife_stance' slots ), the backing of a claim made by a disputant and the warrant ('husband_reasoning' and 'wife_reasoning' slots) for each claim are supported. Both the data and warrant then contribute to support the claim presented at the negotiation.

The following lists the specifications for value slots in one of the frames used, the percentage_frame.

- Case_no: A number indicating the case number this argument pertains to. Together with the Case_arg slot, it allows tracking of arguments by their case numbers.

- Case_arg: Allows entry of the current argument number, which is in numeric sequence with other arguments for the same case. Argument sequencing can gauge whether a certain argumentative sequence was more successful than another. This field allows an independent existance of an argument, of particular interest in the retrieval of custody arguments.

- Total_value: Gives the total value of the common pool to be distributed. This figure is used in the final percentage split calculation. The system assumes entry of a single figure to represent the total common pool value.

- Husband_stance: Stores the percentage split the husband wishes to negotiate by. This figure represents the percentage of monetary advantage from the common pool. These stances, as are the associated reasoning slots, are only valid for this single argument.

- Wife_stance: Stores the percentage split the wife wishes to negotiate by. This figure represents the percentage of monetary advantage from the common pool. 
- Husband_reasoning and Wife_reasoning: Represent reasons (or the backing to a claim) the parties provide to explain why their particular stance has been taken up. These system generated reasons aim to reflect common social attitudes towards current positions.

- Reasons stored as options are as follows:

- Children need financial support

- My homemaker role has contributed substantially

- My income was considered the primary source

- To acheive an equal split.

- Solution: The solution to the negotiation will be stored as a numeral, space, numeral to indicate the percentage split between husband and wife respectively. Solutions are retrieved by the case based reasoner directly from this slot. New arguments store their solution whether successful or not.

- Accepted: If the proposed settlement was rejected, then this slot will store a 'no', otherwise it will store a 'yes'. This slot value is retrieved by the case based reasoner to ascertain whether the dispute has been resolved by this argument or not.

- Comment: Stores further information about the argument, usually concerning its placement in the scheme of other arguments pertaining to the same case. Hence, it may contain the string 'next_arg_3', indicating the next argument in the current sequence is argument 3. This slot is not always assigned, depending on the success of the stored case.

\section{System architecture}

Family_Negotiator has been developed and installed on an IBM PC, under Windows. The current installation has provided for extensive testing of the research developed system. Coding techniques have included use of procedural and object oriented ideas to achieve a modular implementation, consisting of some 20 pages of code.

Family_Negotiator requires the user to enter as input, among other information, the issues yet to be resolved. If the parties do not agree on which issues should be dealt with first, the basic case base is consulted and a relevant adapted plan is retrieved and presented to the parties. This process ensures the disputants have complete control over the manner in which negotiations proceed, an important facet of human negotiation.

Once a plan for negotiation has been agreed to, negotiations can commence. Each issue is dealt with separately by different modules. After each issue has been negotiated successfully, or a failure has been recorded, the next issue commences. This cycle comes to an end when all issues have been dealt with by Family_Negotiator. If an issue has been abruptly ended by the parties, a failure is recorded in the comments slot of the case, enabling its retrieval by later cases. 


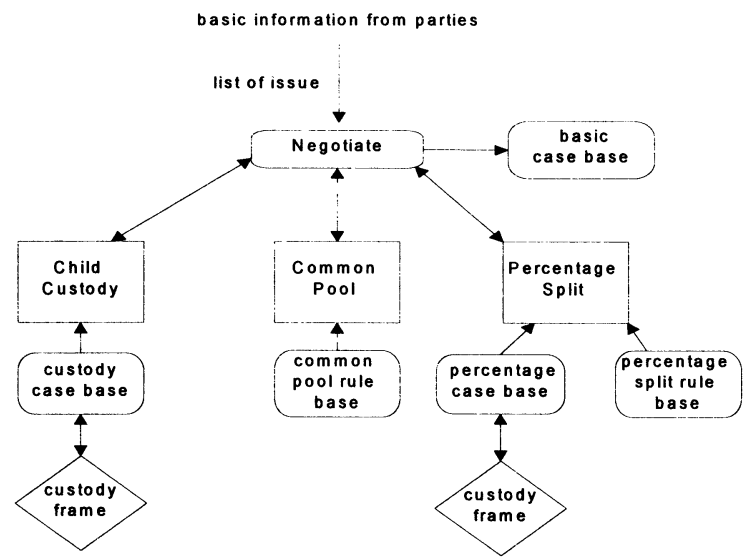

Figure 1 Family_Negotiator's use of Artificial Intelligence paradigms across modules.

Figure 1 distinguishes the three main modules to family law negotiation. Wherever case based reasoning is employed; this is indicated by connection to the module by a case base and the frame responsible for retrieval of cases. If rule based reasoning is used, this is so indicated by linking the module to a specific rule base. The negotiate procedure is responsible for calling the relevant modules and marking the end of an argument. There is no interaction between the three modules, as each has access to their own cases and rule base, allowing the program to achieve a modular architecture which makes program maintenance easy to perform.

\section{Determining the contents of the common pool}

Rule based reasoning is employed in the module dealing with determining the contents of the final common pool. The contents have been classified into several classes, including real estate, chattels, business assets, choses in action and vested interests. The rules implemented in the system represent the common heuristics applicable to the determination of the common pool. One example which stipulates the inclusion of savings accumulated while the couple have been married follows:

IF parties have accumulated savings before separation THEN include in the Common Pool.

In this case, the system will ask the disputants separately if they have accumulated savings during period before seperation. If the party responds 'yes', then these savings are to be included in the common pool.

Rules considered relevant to the domain have become universally accepted heuristics for common pool determination (Zeleznikow and Stranieri, 1995). Hence the inclusion of an overall heuristic which states inclusion into the common pool of all property acquired during the marriage as well as any future acquirement of finances that is currently foreseeable, is justified.

The rule base, consisting of nine if then else constructs, operates using the forward chaining algorithm. The firing of rules commences and continues until a rule with a 
consequent of non inclusion in the common pool is fired, or the rule based reasoning mechanism has reached the end of traversing all rules (in which case the system assumes the item is to be included in the common pool).

\section{Determining percentage splits}

Percentage splits of the common pool value are controlled by hybrid rule based and case based reasoning, in which the rules, representing heuristics and 'norms', are fired foremost. If solutions obtained from the rule base are refused by the parties as a solution to the negotiation, then the case based reasoner retrieves another answer. Solutions are represented in a percentage format, allowing the total value of the common pool be distributed, as opposed to distribution by item. In the rule base, several heuristics are fired to either attain settlement immediately, or to try to persuade the parties to revise their claims, as the following example rules demonstrate.

If children are involved AND

custody of children has been decided, THEN

the party with custody has rights to a greater percentage.

If income of husband is greater than income of wife THEN

wife is entitled to a greater percentage.

ELSE husband is entitled to a greater percentage.

If the couple accept one of the above recommendations, a facililty to revise their percentage claims is presented. If their revised figures add to 100 , then this issue has been successfully negotiated. If not, case based reasoning is attempted, in a similar manner to that performed by the child custody module.

\section{Child custody}

This module is a pure case based reasoner, in which failures and successes are all recorded, to be retrieved during a negotiation session. As with most case based reasoners, it attempts to solve a current problem by referring to its case base. It does not, however, require adapted solutions to ensure they correctly conform to the present case. In the majority of cases, the same issues of child custody, property settlement and percentage dividend settlements are presented as the issues to be resolved. Hence, given the confines of the domain, we do not believe it necessary to use adaptation.

The contents of the case base is the primary source of family law decisions and therefore needs to be extensive and properly indexed. Family_Negotiator has 25 cases in its starting custody case base. The following is an extract of the case base.

case_no 1, case_arg 1, no_children 2, name_of_child Mark, age_of_child 5, childs_wish , husband_stance Wants custody, wife_stance Wants custody, husband_reasoning Close relationship with Child, wife_reasoning I am able to be home more often, solution custody_to_wife, accepted yes, comment.

Once all the cases have been read into memory, the program tries to find the closest matching case to the current case presented. The retrieval algorithm 'Nearest Neighbour' (Kolodner, 1993) is utilised by all case based reasoners in the system. The algorithm's primary focus is to pattern match slot values with the corresponding values of the current 
case. All attributes which are matched against by the case based reasoner have an importance value attached to it. This value is representative of the attributes importance or contribution to retrieving the best solution currently available in the case base. These have value ranges between 0 and 1, where an importance_value of 0 indicates no importance, and a value 1 indicates the greatest grading of importance given to an attribute. For example, in the child custody component, importance_no_children $=0.3$, and importance_husband_reasoning $=$ 1. These figures were calculated from data collated from the Melbourne Association of Family Lawyers.

The retrieval mechanism in this component uses the above importance values to ascertain which case in the case base is most similar to the current negotiation. An example follows:

Taking the attribute no_children,

$$
\text { no_children_current }=1 \text { and no_children_case }=2
$$

The difference between these values is calculated to determine how close the case is to the currently presented situation. The difference between these is given in a double integer format, hence for this example will be $\bmod (10-20)=10$.

Since the difference is 10 , the difference_weight generated by function difference_weights() $=0.9$. If the difference was between 10 and 20 then a value of 0.8 is added to the total weight of the case, and so on.

If the slot is non numeric in value, and a match exists, its importance_value is added to the total_weight, else no value is added.

Total_weight $=0($ since case is first $)+0.9 *$ importance_no_children.

$$
=0.18
$$

The total weight is made up of by the summation of all slot value comparisions for the case. After the cummulative total_weight is finalised, the individual difference values used in the case retrieval mechanism are added together. The above total is hence divided by this summation $(0.9+\ldots)$.

Once all cases in the case base have been traversed, a set of weight scores are generated. The case whose weight is greatest in value is then considered the most similar case to that currently presented to the system, and consequently its case answer is retrieved and presented to the user for evaluation.

An evaluation of Family_Negotiator was performed under the supervision of domain expert Dr. Richard Ingleby, Adjunct Professor of Family Law, University of Melbourne and a practising barrister (trial attorney). The system was trialled by the Melbourne Association of Family Lawyers who received it warmly. 


\section{CONCLUSION}

Our research has indicated the need for negotiation support in family law, and has presented a solution, through the implementation of Family_Negotiator. The hybrid paradigm employed has enabled the system to be flexible in tackling important issues, choosing paradigms which best models the current issue in dispute. As a decision support tool, Family_Negotiator gives parties an insight into past successes and failures, and thus enabling successful outcomes to the negotiation task at hand.

\section{REFERENCES}

Bellucci, E. and Zeleznikow, J. (1996) Family_Negotiator: using multi-modal reasoning to support negotiation. To appear in proceedings of Asia Pacific DSI Conference 1996.

Fisher, R. , Ury, W. and Patton, B. (1994) Getting to Yes. Century Business, Sydney, 5-11.

Kolodner, J. (1993) Case-based Reasoning. Morgan Kaufmann Publishers, California, 354 358.

Toulmin, S. (1958). The uses of argument. Cambridge, Cambridge University Press.

Zeleznikow, J. and Hunter, D. (1994) Building Intelligent Decision Support Systems through the use of Multiple Reasoning Strategies.

Zeleznikow, J. and Stranieri, A. (1994) Reasoning in Open textured Domains: Benefits of integrating multiple reasoning strategies.

Zeleznikow, J., Meersman, R., Hunter, D. and van Helvoort, E. (1995) Computer tools for aiding legal negotiation. ACIS95 - Sixth Australian Conference on Information Systems, Curtin University of Technology, Perth, Western Australia 231-251.

Zeleznikow, J. and Stranieri, A. (1995) The Split-Up system: Integrating neural networks and rule-based reasoning in the legal domain. Proceedings of Fifth International Conference on Artificial Intelligence and Law, New York, ACM Press, 185-194.

\section{BIBLIOGRAPHY}

Emilia Bellucci is a Ph.D. student at La Trobe University, Australia. She is currently working on building intelligent legal negotiation systems.

Dr. John Zeleznikow has written over 50 papers on Artificial Intelligence and Databases and has co written a book on building intelligent legal systems. He will be the General Chairman of the 1997 International Conference of Artificial Intelligence and Law. 